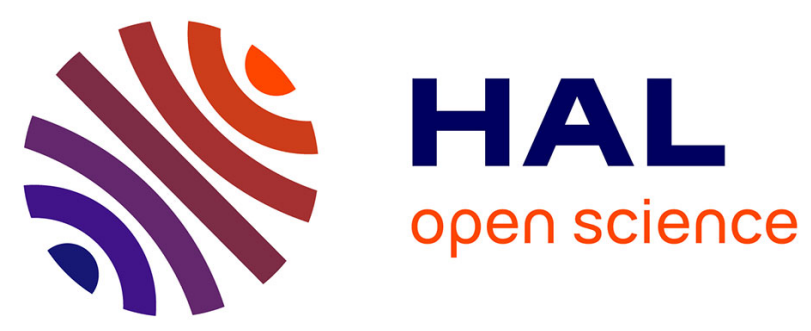

\title{
Optic neuropathy, cardiomyopathy, cognitive disability in patients with a homozygous mutation in the nuclear MTO1 and a mitochondrial MT-TF variant
}

\author{
Majida Charif, Salah Mohamed Cherif Titah, Agathe Roubertie, Valerie
}

Desquiret-Dumas, Naig Gueguen, Isabelle Meunier, Jean Leid, Frédéric

Massal, Xavier Zanlonghi, Jacques Mercier, et al.

\section{To cite this version:}

Majida Charif, Salah Mohamed Cherif Titah, Agathe Roubertie, Valerie Desquiret-Dumas, Naig Gueguen, et al.. Optic neuropathy, cardiomyopathy, cognitive disability in patients with a homozygous mutation in the nuclear MTO1 and a mitochondrial MT-TF variant. American Journal of Medical Genetics Part A, 2015, 167 (10), pp.2366-2374. 10.1002/ajmg.a.37188 . hal-01756203

\section{HAL Id: hal-01756203 \\ https://hal.umontpellier.fr/hal-01756203}

Submitted on 12 Sep 2018

HAL is a multi-disciplinary open access archive for the deposit and dissemination of scientific research documents, whether they are published or not. The documents may come from teaching and research institutions in France or abroad, or from public or private research centers.
L'archive ouverte pluridisciplinaire HAL, est destinée au dépôt et à la diffusion de documents scientifiques de niveau recherche, publiés ou non, émanant des établissements d'enseignement et de recherche français ou étrangers, des laboratoires publics ou privés. 


\title{
Optic Neuropathy, Cardiomyopathy, Cognitive Disability in Patients with a Homozygous Mutation in the Nuclear MT01 and a Mitochondrial MT-TF Variant
}

\author{
Majida Charif, ${ }^{1,2,3}$ Salah Mohamed Cherif Titah, ${ }^{4}$ Agathe Roubertie, ${ }^{5}$ Valérie Desquiret-Dumas, ${ }^{6, ?}$ \\ Naig Gueguen, ${ }^{6,7}$ Isabelle Meunier, ${ }^{1,2,3,4}$ Jean Leid, $^{8}$ Frédéric Massal, ${ }^{8}$ Xavier Zanlonghi, ${ }^{9}$ \\ Jacques Mercier, ${ }^{10,11}$ Eric Raynaud de Mauverger, ${ }^{10,11}$ Vincent Procaccio, ${ }^{6,7}$ \\ Bénédicte Mousson de Camaret, ${ }^{12}$ Guy Lenaers, ${ }^{1,2,3}$ and Christian P. Hamel ${ }^{1,2,3,4 *}$ \\ ${ }^{1}$ INSERM U1051, Institute for Neurosciences of Montpellier, Montpellier, France \\ ${ }^{2}$ Université Montpellier 1, Montpellier, France \\ ${ }^{3}$ Université Montpellier 2, Montpellier, France \\ ${ }^{4} \mathrm{CHRU}$ Montpellier, Centre de Référence Maladies Sensorielles Génétiques, Montpellier, France \\ ${ }^{5}$ CHRU Montpellier, Service de Neuropédiatrie, Montpellier, France \\ ${ }^{6}$ Département de Biochimie et Génétique, CHRU Angers, Angers, France \\ ?UMR CNRS 6214-INSERM U1083, Université Angers, Angers, France \\ ${ }^{8}$ Ophthalmology, Eye Center, Pau, France \\ ${ }^{9}$ Clinique Sourdille, Visual Explorations, Nantes, France \\ ${ }^{10}$ CHRU Montpellier, CERAMM, Montpellier, France \\ ${ }^{11}$ INSERM U1046, Médecine et Physiologie Expérimentale du Cœur et des Muscles, Montpellier, France \\ ${ }^{12}$ Service des Maladies Héréditaires du Métabolisme, Centre de Biologie et de Pathologie Est, CHU Lyon, Bron, France
}

We report on clinical, genetic and metabolic investigations in a family with optic neuropathy, non-progressive cardiomyopathy and cognitive disability. Ophthalmic investigations (slit lamp examination, funduscopy, OCT scan of the optic nerve, ERG and VEP) a disclosed mild or no decreased visual acuity, but pale optic disc, loss of temporal optic fibers and decreased VEPs. Mitochondrial DNA and exome sequencing revealed a novel homozygous mutation in the nuclear MTO1 gene and the homoplasmic m.593T $>$ G mutation in the mitochondrial MT-TF gene. Muscle biopsy analyses revealed decreased oxygraphic Vmax values for complexes I+III + IV, and severely decreased activities of the respiratory chain complexes (RCC) I, III and IV, while muscle histopathology was normal.
Fibroblast analysis revealed decreased complex I and IV activity and assembly, while cybrid analysis revealed a partial complex I deficiency with normal assembly of the RCC. Thus, in patients with a moderate clinical presentation due to $M T O 1$ mutations, the presence of an optic atrophy should be considered. The association with the mitochondrial mutation $\mathrm{m.593T}>\mathrm{G}$ could act synergistically to worsen the complex I deficiency and modulate the MTO1-related disease.

Key words: optic neuropathy; cardiomyopathy; cognitive disability; mitochondria; mtDNA; respiratory chain; MTO1; mitochondrial tRNA

Majida Charif and Salah Mohamed Cherif Titah contributed equally to this work.

Conflict of interest: none.

Grant sponsor: French Ministry of Health; Grant sponsor: Union Nationale des Aveugles et Déficients Visuels; Grant sponsor: Ouvrir Les Yeux.

*Correspondence to:

Christian P. Hamel, INSERM U. 1051, Institut des Neurosciences de Montpellier, Hôpital Saint-Eloi, BP 74103, 80, rue Augustin Fliche, 34091

Montpellier Cedex 5, France.

E-mail: christian.hamel@inserm.fr 


\section{INTRODUCTION}

Mitochondriopathies are among the most frequent inherited diseases. Clinical presentation can be restricted to a single organ like in the Leber hereditary optic neuropathy, or be syndromic affecting neurological (brain, sense organs, peripheral nervous system), muscular (heart and skeletal muscles), endocrinological and renal-liver systems [Jackson et al., 1995; Munnich et al., 1996; Chinnery et al., 1997]. In many cases, mitochondriopathies are due to mutations in nuclear genes, and it is anticipated that several hundreds of nucleargenes cause mitochondrial diseases [Rotig et al., 2004; Wallace et al., 2010]. Mitochondriopathies are also be due to alterations in the mtDNA (prevalence 1/10,000), being found in the 13 genes encoding subunits of the respiratory chain complexes (RCC), 22 tRNAs and two rRNAs [Chinnery et al., 2012]. The mitochondriopathies caused by mutations in the mitochondrial DNA include MELAS (Myopathy, Encephalopathy, Lactic Acidosis and Stroke like-episodes) associated in $80 \%$ of cases to the m.3243A $>$ G mutation in the MT-TL1 gene coding the tRNA ${ }^{\text {Leu }}$, MERRF (Myoclonic Epilepsy with Ragged Red Fibers) associatedin $80 \%$ ofthecasestothem. $8344 \mathrm{~A}>$ Gmutation [Schmiedel et al., 2003], LHON (Leber Hereditary Optic Neuropathy) associated to the the three primary mutations $\mathrm{m} .3460 \mathrm{~A}>\mathrm{G}, \mathrm{m} .11778 \mathrm{~A}>\mathrm{G}$, and m.14484T $>C$ accounting for $95 \%$ of the cases [Tonska et al., 2010].Leigh syndrome (developmentaldelay, seizures, lacticacidosis, hypotonia, ataxia, optic atrophy, retinitis pigmentosa, ophthalmoplegia, ptosis) is associated to mutations in nuclear or mtDNAencoded subunits of various RCC complexes [Koopman et al., 2013].

A novel form of mitochondrial disease associating hypertrophic cardiomyopathy and lactic acidosis was recently described [Ghezzi et al., 2012; Baruffini et al., 2013]. This mitochondriopathy is caused by mutations in the MTO1 gene, encoding the mitochondrial translation optimization 1 (MTO1) protein. Patients either undergo severe disease course and early death associated to cardiac failure, or have milder presentation controlled by drug treatment. In one case in an adult patient, moderate bilateral optic neuropathy was reported in association with the lack of ocular fixation, weakness and bradycardia [Baruffini et al., 2013]. In this study, we report on two siblings who presented a multisystemic disorder associating optic atrophy, cardiomyopathy, cognitive disability and seizures due to a novel homozygote mutation in the nuclear MTO1 gene. In addition, the patients and their mother carried a homoplasmic m.593T $>\mathrm{G}$ transversion in the mitochondrial $M T$ $T F$ gene, encoding the tRNA ${ }^{\text {phe }}$. Muscle biopsy, skin fibroblasts and cybrid cells revealed significant impairments of the mitochondrial RCC, suggesting that the mitochondrial variant might contribute to the clinical presentation of the disease.

\section{MATERIALS AND METHODS \\ Clinical Examination}

Visual acuity was determined and the visual field was tested by Goldmann perimetry. Full-field electroretinography (ERG) was performed according to the guidelines of the International Society for Clinical Electrophysiology of Vision, using a Ganzfeld apparatus (Ophthalmologic Monitor, Métrovision, Pérenchies, France). Color fundus imaging (Topcon Imagenet, Ophthalmic Imaging Systems, Japan) and autofluorescence imaging (Heidelberg Retina
Angiograph (HRA) 2, Heidelberg Engineering, Dossenheim, Germany) documented retinal findings. The macula was analysed using the optical coherent tomograph (Stratus OCT3, Carl Zeiss Meditech, Inc., Dublin, California, USA-Retinal Thickness Map512 pixels). Visual Evoked Potentials were recorded by using a Sirius Galileo instrument (Esaote Biomedica, Florence, Italy).

Affected patients had standard cardiac examination and cardiac echography. They underwent an incremental maximal exercise test on a treadmill (LE 200 CE Jaegger, Hoechberg, Germany). During the test, oxygen uptake, carbon dioxide production, and minute ventilation were measured breath by breath using an open-circuit metabolic cart (Oxycon Pro, Jaegger). Each stage consisted of a 1min exercise period and increased by $10 \%$ of theoretical Pmax $/ \mathrm{min}$.

\section{Metabolic Investigations}

\section{Oxygen consumption and enzymatic measurements on skeletal}

muscle. Vastus lateralis muscle biopsies were taken by the percutaneous Bergström technique after local anesthesia (Xylocaine). The muscle samples were divided into two portions: one was placed in a standard medium for histoenzymological analysis, the other one was immediately placed in an ice-cold relaxing solution, at $160 \mathrm{mM}$ (potassium methanesulfonate), $\mathrm{pH}$ 7.1, containing $10 \mathrm{mM}$ EGTA-calcium buffer (free $\mathrm{Ca}^{2+}$ concentration: $100 \mathrm{nmol} / \mathrm{l}), 20 \mathrm{mM}$ imidazole, $3 \mathrm{mM} \mathrm{KH} \mathrm{PO}_{4}, 1 \mathrm{mM} \mathrm{MgCl}$, $20 \mathrm{mM}$ taurine, $0.5 \mathrm{mM}$ DTT, $5 \mathrm{mM} \mathrm{MgATP}$, and $15 \mathrm{mM}$ phosphocreatine, and used for the in situ respiration studies [Saks et al., 1998]. The fiber bundles were separated with sharp-ended needles, leaving only small areas of contact, and were incubated in $1 \mathrm{ml}$ of the relaxing solution $\left(4^{\circ} \mathrm{C}\right)$ containing $50 \mu \mathrm{g} / \mathrm{ml}$ saponin for $30 \mathrm{~min}$ with continuous stirring. To completely remove saponin, the fibers were washed with continuous stirring with relaxing solution for $10 \mathrm{~min}\left(4^{\circ} \mathrm{C}\right)$. To remove free ATP, they were then washed for $2 \times 5 \mathrm{~min}$ at $4^{\circ} \mathrm{C}$ with the oxygraph solution, similar to the relaxing solution, except that MgATP and phosphocreatine were replaced by $2 \mathrm{mM}$ malate, $3 \mathrm{mM}$ phosphate, and $2 \mathrm{mM}$ fatty acid-free bovine serum albumin, $\mathrm{pH}$ 7.1. After washing, the fibers were stored on ice in oxygraph solution until determination of mitochondrial respiration activities [Thomas et al., 2004].

Mitochondrial metabolic function was assessed ex vivo using high-resolution respirometry, in permeabilized vastus lateralis 
muscle fibers, as previously described [Sirvent et al., 2012]. Respiratory chain complex (RCC) activities were measured in a $800-\mathrm{g}$ supernatant of crude muscle homogenates as described [Mousson De Camaret et al., 2007]. Adenosine triphosphate (ATP) measurements and ATP synthesis were performed according to Ouhabi and colleagues [Ouhabi et al., 1998].

Fibroblast cultures and cybrid experiments. Fibroblast primary cultures obtained from patient skin biopsies were cultured in RPMI 1,640 medium supplemented with glutaMAX (Invitrogen, Cergy Pontoise, France), 10\% fetal bovine serum (FBS), $100 \mu \mathrm{g} / \mathrm{ml}$ sodium pyruvate and $50 \mu \mathrm{g} / \mathrm{ml}$ uridine in a $5 \% \mathrm{CO}_{2}$ atmosphere at $37^{\circ} \mathrm{C}$. Mitochondrial network morphology and mitochondrial membrane potential $(\Delta \Psi \mathrm{m})$ were explored as described elsewhere [Olichon et al., 2007]. Cybrid patient cells were generated with a method adapted from Ishikawa and Hayashi (17). Briefly, patient fibroblasts were enucleated by an actinomycin D treatment $(0.5 \mu \mathrm{g} /$ $\mathrm{ml}$ ) and fused to Rho ${ }^{0} 143 \mathrm{TK}^{-}$cells with PEG (MW 1500, Roche Applied Science). The medium was replaced 2 days post fusion with selective medium (DMEM, 10\% dialysed Foetal Bovine Serum, $30 \mu \mathrm{g} / \mathrm{ml}$ 5-bromo-2-deoxyuridine). Cybrid clones were collected and were subsequently expanded during several passages. Enzymatic activities of respiratory chain complex I, II, and IV (Table IV) were measured in control and patient fibroblasts and cybrids according to Loiseau et al. [2007].

Blue native PAGE (BN PAGE). Mitochondria-enriched extracts were obtained from fibroblasts and cybrids by differential centrifugation and resuspended at $5 \mathrm{mg} / \mathrm{ml}$ in $1.5 \mathrm{M}$ 6-aminocaproïc acid, $75 \mathrm{mM}$ Bis Tris at pH 7.0 and antiprotease (Roche). N-dodecyl$\beta$-D-maltopyranoside ( $3 \mathrm{~g} / \mathrm{g}$ of mitochondrial protein) was added for ten minutes to solubilize complexes in monomeric form and supercomplex assembly. After 20 min of centrifugation at $10,000 \mathrm{~g}$, the supernatant was collected and added to the loading buffer (Coomassie brilliant blue G 250 5\%, 6-aminocaproïc acid 750 mM, EDTA $0.5 \mathrm{mM}$ and Bis Tris $50 \mathrm{mM}$ at pH 7.0). $40 \mu \mathrm{g}$ of proteins were loaded on blue native PAGE 3-12\% Bis-Tris gel (Invitrogen) and separated at 150 volts. Following the transfer to PVDF membranes (Amersham) and saturation with $10 \%$ in non-fat milk dissolved in TBS-Tween $0.1 \%$, the presence of complex I, II, III, and IV were assessed using antibodies against NDUFA9 and NDUFB6, SDHA, III core 2 and COX I respectively (Mitosciences) and revealed by anti-mouse IgG horseradish peroxidase linked antibody (1/10,000 Sigma).

DNA sequencing. Genomic DNA was extracted from blood, fibroblasts and muscle samples by standard methods. Total mitochondrial DNA from the proband was PCR-amplified in eight overlapping fragments and directly sequenced with the BigDye terminator chemistry as described elsewhere [Nochez et al., 2009]. Sequence data were analyzed using Seqscape (Life Technologies, Carlsbad, California) and compared with the Cambridge reference sequence. Whole-exome sequencing (WES) was performed using SureSelect Human All Exon Kits Version 3 in-solution enrichment methodology (Agilent, Santa Clara, CA) by Integragen (France).

Gene references. The MTO1 gene is located on chromosome $6 \mathrm{p} 13$ and referenced in NCBI OMIM database as ${ }^{\star} 614667$. The MTO1 transcript is referenced as ENST00000415954, and the inferred 692 amino-acid protein sequence as CCDS47452.1 in Ensembl. The mitochondrial MT-TF gene is referenced in NCBI OMIM database as ${ }^{\star} 590070$ and as ENSG00000210049 in Ensembl.
Ethical issues. This research followed the Declaration of Helsinki.

Informed consent to perform genetic and molecular analysis was obtained from each patient. The authors confirm that they are in compliance with their Institutional Review Boards. The Department of Ophthalmology of the Hospital of Montpellier has the authorization \#11018S from the French Ministry of Health for biomedical research in the field of physiology, pathophysiology, epidemiology, and genetics in ophthalmology.

\section{RESULTS \\ Clinical Findings}

The family was composed of two related healthy parents and of their two affected adult children (Fig. 1A), without offspring. Parents and their ascendants had no known neuro-sensorial or cardiac disease.

Patient II-1 had failure to thrive in early infancy. Later on, mild cognitive disability was noticed. Yet, she was able to read and became a factory worker. At age 7, because of sudden weakness after walking, she was found to have left concentric ventricular hypertrophic cardiomyopathy with the septum at $13 \mathrm{~mm}$ (normal $=7$ $\mathrm{mm}$ ) and inferior wall at $13 \mathrm{~mm}$ (normal $=7 \mathrm{~mm}$ ). She had normal ventricular kinetics with rejection fraction at $60 \%$. Arterial and intra-cardiac pressures were also normal indicating the absence of cardiac insufficiency. Since then, she had been treated with $160 \mathrm{mg} /$ day propranolol. Echocardiographic follow ups indicated that the cardiomyopathy was stable. At age 8 , she experienced childhood absence epilepsy. The EEG showed, on a normal baseline activity, bilateral, synchronous, generalized spike waves, exacerbated by eye closure, that later organized on a more parietotemporal focus. She was treated with $1 \mathrm{~g} /$ day valproic acid until age 18, and lamotrigine treatment had to be introduced at age 24 because of seizure relapse. Neurological examination was normal. Blood cell counts and usual biological parameters were normal. She had no diabetes. Auditory function was normal. She had kyphoscoliosis since early childhood. Surgical arthrodesis was performed at age 16. Brain and optic pathway MRI were performed several times and were normal. At age 22, she complained of low vision. She had been moderately photophobic since early childhood. Visual acuity was decreased to 20/30 on the right eye and 20/40 on the left eye with moderate alteration of color vision but no impairment of the peripheral visual field. Loss in visual acuity progressed and she stopped driving at 26. At age 30, her best corrected visual acuity had worsened to $20 / 100$ on the right eye and $20 / 70\left(-050 ; 160^{\circ}\right)$ on the left eye. Photomotor reflexes and intraocular pressure were normal. Fundus examination revealed atrophic optic discs (Fig. 1B), whereas the macula, peripheral retina and vessels were normal. OCT-3 showed significant reduced thickness of the temporal nerve fiber layer and visual field testing revealed a caecocentral scotoma in the right eye and widening of the blind spot in the left eye (not shown). The electroretinogram in photopic condition was normal. The pattern visual evoked potentials (VEPs) showed a P100 reduced amplitude with subnormal latency (Fig. 1E). Amplitudes of the flash VEPs were also decreased but latencies were normal. 
A

$1-1$

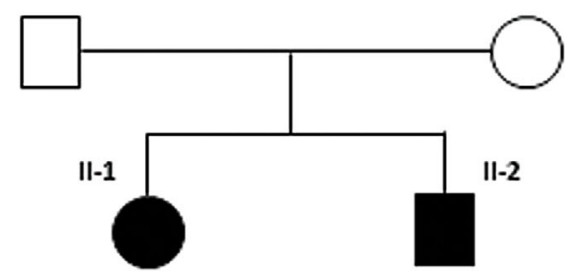

C patient II-2

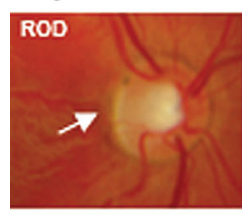

LOD
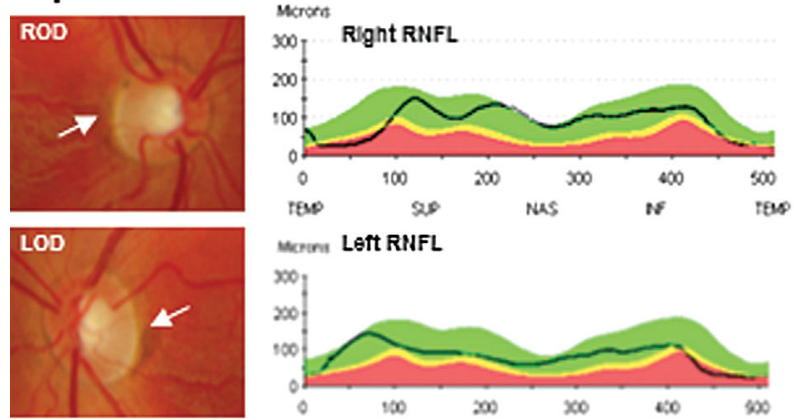

Hern Left RMFL

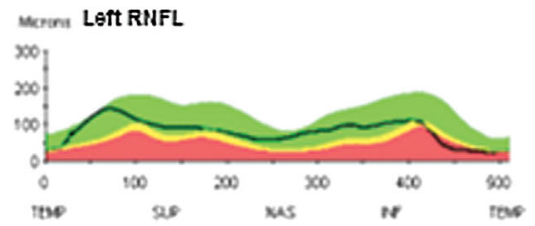

B patient II-1
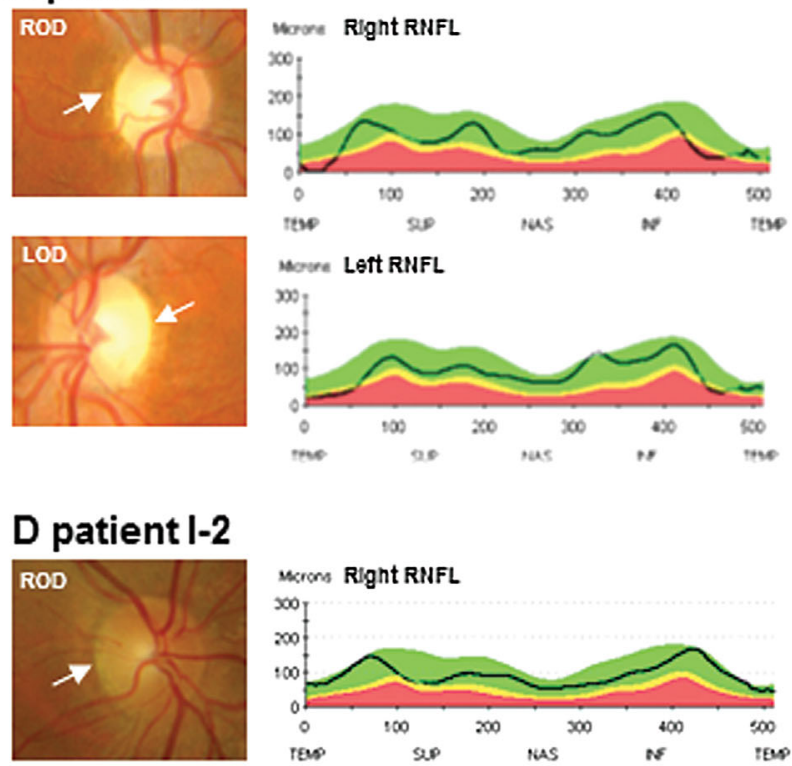

LOD

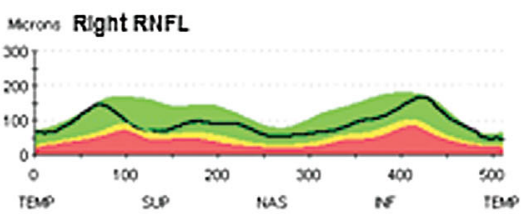

merim Left RNFL

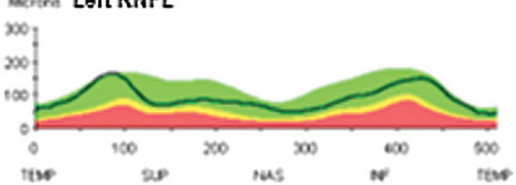

E

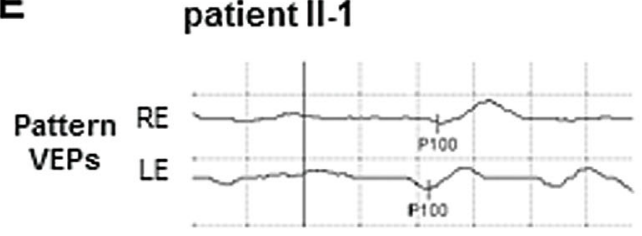

Flash

VEPs

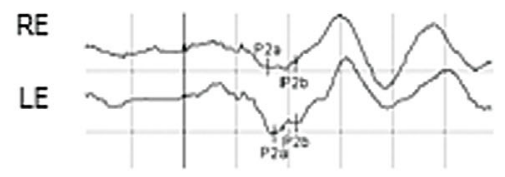

patient II-2
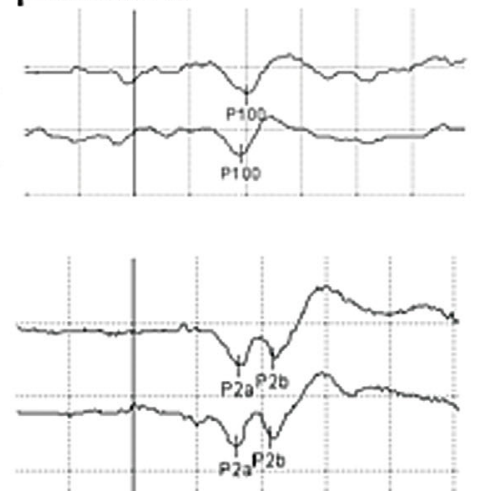

normal

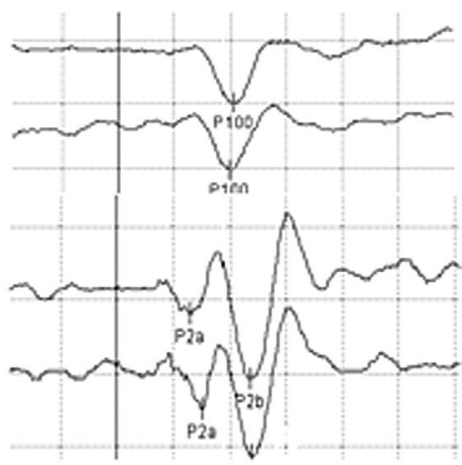

FIG. 1. Ophthalmological findings. A: pedigree of the family. B, C, D: for each of the three patients, color photographs of the optic disc (left panels) in each eye (ROD: right optic disc; LOD: left optic disc) are shown. White arrows indicate the temporal side of the optic discs which are pale in patients II-1 and II-2. On the right panels, the OCT-3 scans measuring the retinal nerve fiber layer (RNFL) are shown for each eye. E, pattern and Flash VEPs for patients II-1 and II-2, and for a normal individual, are shown. VEPs amplitudes of both patients are reduced.

Patient II-2 had a more severe cognitive disability than his older sister. He was never able to read and he worked in a factory for mentally disabled patients. At age 5 , he complained of fatigue and a left ventricular hypertrophic cardiomyopathy (septum $10 \mathrm{~mm}$, inferior wall $10 \mathrm{~mm}$ ) was discovered. He had normal $63 \%$ rejection fraction without cardiac insufficiency. Since then, he was treated with propranolol and he currently receives $160 \mathrm{mg}$ /day. The size of both septum and inferior wall increased to $12 \mathrm{~mm}$ but the rejection fraction remained normal to $62 \%$. He had moderate asthma and dorsal kyphosis which did not require surgery. He had no diabetes. He complained occasionally of muscle pain, but there was no muscle atrophy and the electromyogram and nerve conduction were normal. Neurological examination was normal.

This patient had no history of visual symptoms. At age 28 , his visual acuity was $20 / 20$ on both eyes with +0.50 . However, fundus examination revealed a moderate temporal pallor of both optic 
discs indicating that he had optic atrophy (Fig. 1C). OCT-3 confirmed a reduced thickness of the temporal nerve fiber layer in both eyes, but visual field testing could not be performed because of cognitive disability. The electroretinogram in photopic conditions was normal. Pattern VEPs showed reduced P100 amplitudes and normal latencies (Fig. 1E). Amplitudes of the flash VEPs were also decreased but latencies were normal.

I-2 mother was 55 . She had no cardiomyopathy or scoliosis and was mentally normal. Visual acuity was $20 / 20$ in both eyes. Fundus showed multiple yellowish deposits of various sizes in mid periphery of the retina. However, the optic discs were normal (Fig. 1D). Normal OCT-3, normal color vision and normal visual field indicated that she had no optic atrophy.

I-1 father had no disability. His eye examination was normal.

The association of optic neuropathy with cognitive disability, seizures, myopathy (cardiomyopathy, scoliosis) in both siblings prompted investigations towards a mitochondrial disorder.

\section{Exercise Testing}

Both Patients II-1 and II-2 showed a limited exercise capacity. $\mathrm{VO}_{2}$ max was at $32 \%$ and $28 \%$ of the normal value for Patients II- 1 and II-2, respectively. At rest, serum lactate was very high in Patient II-1 (6.5 mmol/l; normal $<2 \mathrm{mmol} / \mathrm{l})$, while it was normal for Patient II-2. At exhaustion, both patients had a normal lactate/ pyruvate ratio ( 83.6 and 46 for Patients II- 1 and II-2, respectively) while the $\beta$-hydroxybutyrate/acetoacetate ratio was elevated (32 and 13.1 for Patients II-1 and II-2, respectively) indicating limited OXPHOS capacity.

\section{Laboratory Investigations}

Biochemical and histological analysis of muscle biopsies. Oxygraphy analysis of permeabilized muscle fibres showed a significant reduction of complexes I + III + IV for both patients, while the values for complexes II + III + IV and for isolated IV, were either fully normal for Patient II-1 or at the highest range of normal values for Patient II-2 (Table I), suggesting a predominant defect of mitochondrial respiratory complex I. Histological analyses of the muscle fibres of both patients did not reveal ragged-red fibres nor gross abnormalities. On the crude muscle homogenate, the citrate synthase activity was increased suggesting a mitochondrial proliferation. In both Patients II-1 and II-2, enzymatic activities of the respiratory chain complexes I, III and IV were severely decreased, whereas the complex II activity was normal. The values of ratios between complex activities supported the defects of complex I, III, and IV (Table II).

Genetic screening. Leukocyte DNA of patient II-1 had none of the three primary or nine secondary mtDNA mutations associated with LHON, nor the m.3243A $>\mathrm{G}$, the m.8993T $>\mathrm{G}$ or the $\mathrm{m} .8993 \mathrm{~T}>\mathrm{C}$ mutations responsible for MELAS, NARP or Leigh syndromes, respectively. There was no mtDNA rearrangement in muscle, and sequencing of POLG, C10ORF2, and ANT1 was negative. Sequence of muscle mitochondrial $12 \mathrm{~S}$ and $16 \mathrm{~S}$ rRNA was normal. However, a search for mutations in the 22 mitochondrial tRNAs identified a homoplasmic variation, $\mathrm{m} .593 \mathrm{~T}>\mathrm{G}$ in the $M T$ TF gene, in both muscle and leukocytes of Patient II-1. Leukocyte mtDNA from the affected brother (II-2) and the unaffected mother (I-2) were also homoplasmic for this sequence variation, while it was absent from the father (I-1) (Fig. 2A). Full sequencing of leukocyte mtDNA of Patient II-1 revealed known mtDNA polymorphisms corresponding to the caucasian haplogroup $\mathrm{H} 1 \mathrm{j}$ with the presence of the $\mathrm{m} .7028 \mathrm{~T}>\mathrm{C}$ and $\mathrm{m} .3010 \mathrm{G}>\mathrm{A}$ polymorphisms, but no other pathogenic DNA mutations (Table III). The m.593T $>\mathrm{G}$ mutation affects the $\mathrm{D}$ loop structure of the tRNA $^{\text {phe }}$ (Figs. 3A and 3B), not highly conserved with a species conservation index estimated at $33 \%$ and is only identified in two individuals from a large dataset of more than 26,850 GenBank sequences from Mitomaster software analysis (http://www.mitomap.org/bin/view.pl/MITOMASTER/WebHome). Interestingly both mtDNA sequences belong to the same mitochondrial haplogroup $\mathrm{H} 1 \mathrm{j}$ except for the presence of a private variant at position $\mathrm{m} .8950 \mathrm{G}<\mathrm{A}$ in our family.

Because the mother of both patients was asymptomatic, we also searched for nuclear mutations by whole exome sequencing of the two affected patients. We found that the two Patients II-1 and II-2 were homozygous for the missense mutation c.1510C $>\mathrm{T}$ in the MTO1 gene, each parent being heterozygous (Fig. 2B). This mutation leads to the p.R504C amino acid change in a highly conserved domain from human to bacteria (Fig. 2C), suggesting its pathogenicity.

Patient fibroblasts and cybrid analysis. Skin fibroblasts of Patient II-1 showed normal mitochondrial network and mitochondrial membrane potential (not shown). However, they displayed greater susceptibility to staurosporine-induced apoptosis, with $49 \% \pm 7.4 \%$ of apoptotic nuclei in the patient, versus

TABLE I. Oxygraphic Analysis of Permeabilized Muscle Fibres

\section{Analyzed pathway}

Substrates

Patient II-1

Patient II-2

Reference values 7.2 (3-

14)
Krebs cycle $+\mathrm{Cl}$, Cll, CIV

Glutamate

$1.1^{*}$

1.3

$7.2(3-14)$

$\mathrm{PDH}+$ Krebs cycle $+\mathrm{Cl}$, CII, CIV

Pyruvate

1.0

1.0

$7.3(3.7-13)$
CII, CIII, CIV

Rotenone + succinate

4.2

14

$5.4(2.1-14)$
CIV

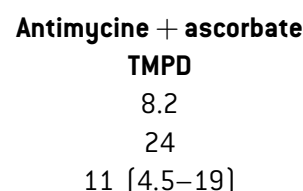

*All values are Vmax given in $\mu \mathrm{mol} \mathrm{O}_{2} / \mathrm{min} / \mathrm{g}$. Reference values are given as a median (range). 
TABLE II. Respiratory Chain Complex Activities in Muscle

\section{Complexes}

Complex I (NADH ubiquinone reductase)

Complex II (Succinate ubiquinone reductase)

Complex III (Ubiquinol cytochrome c reductase)

Complex IV (Cytochrome c oxidase

Citrate synthase

Patient II-1
$0.5^{*}$
23.5
40.3
12.2
369.0

Patient II-2

1.1

24.8

59.2

18.2

696.3
Control values $(n=40)$

$13.7(9.8-18.3)$

$19.3(13.8-28.0)$

$118.4(93.1-139.6)$

$75.7(48.5-107.2)$

$188.4(103-304)$

*Activities are given in $\mathrm{nmol} / \mathrm{min} / \mathrm{mg}$ protein and normalized with respect to citrate synthase activity. Control enzyme activities are given as a mean (range).

$34 \% \pm 2.2 \%$ in control cells. Defects of complex I and IV activities with residual activities of $46 \%$ and $57 \%$ of normal values respectively, were found, while complex II activity was normal (Table IV). This paralleled an important decrease of the abundance of $\mathrm{CI}$ and an almost complete disappearance of CIV, as observed by BN PAGE (Fig. 3C). To further test the pathogenic contribution of the m.593T>G mutation to this metabolic phenotype, enucleated fibroblasts from control and patient II- 1 were fused to rho( $(0)$ cells, fully depleted of mtDNA. The analysis of cybrid cells carrying the m.593T $>$ G by BN PAGE revealed a normal composition of the respiratory chain complexes, suggesting that the m.593T $>$ G mutation has no detectable effect on the translation of the mitochondrial encoded proteins from the RCC (Fig. 3C). Nevertheless, the m.593T $>$ G cybrid cells showed a reduced CI enzymatic activity with a residual activity of $45 \%$ of normal value, similar to the CI activity found in fibroblasts, while CIV activity was normal compared to control level.

\section{DISCUSSION}

The diagnosis of mitochondriopathy in the condition presented herein was based on the initial observation of suggestive clinical signs and impaired mitochondrial respiratory chain. We later found that the affected patients were homozygous for a MTO1 mutation, encoding a modifier of the mitochondrial tRNAs, known to cause hypertrophic cardiomyopathy and lactic acidosis. Interestingly, the patients also had a MT-TF mutation at position $\mathrm{m} .593 \mathrm{~T}>\mathrm{G}$.

In mitochondrial disorders, myocardiopathy, childhood-onset absence epilepsy and cognitive disability are frequently observed, in respectively $10 \%, 45 \%$, and $20 \%$ of all cases [Terasaki et al., 2001], as kyphoscoliosis linked to muscle weakness [Munnich et al., 1996]. In the two patients harboring the MTO1 mutation presented in this study, we found the association of myocardiopathy, cognitive disability, kyphoscoliosis, optic neuropathy and epilepsy only in one patient. This corresponds to the clinical presentation recently described in patients with MTO1 mutations [Ghezzi et al., 2012; Baruffini et al., 2013]. In four out of eight patients who reached adolescent or adult stages, all had a combination of hypertrophic cardiomyopathy and lactic acidosis. In addition, two of them also had psychomotor delay, one had encephalopathy and seizures, and the fourth one had muscle weakness. Only one of their two adult patients (19- and 20-year-old) had a visual defect described as a lack of ocular fixation and moderate bilateral optic atrophy. In Patient II-2 (28-year-
A
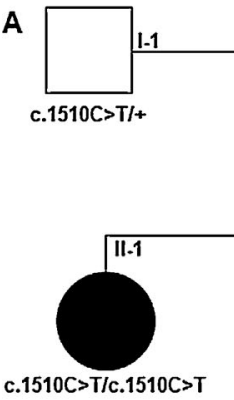
$\mathrm{m} .593 \mathrm{~T}>\mathrm{G}$

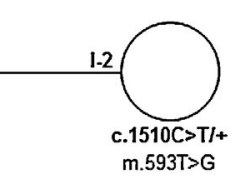

m.593T>G

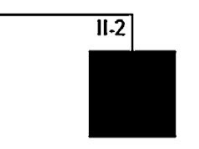

c. $1510 \mathrm{C}>\mathrm{T} / \mathrm{c} .1510 \mathrm{C}>\mathrm{T}$ m.593T>G
B

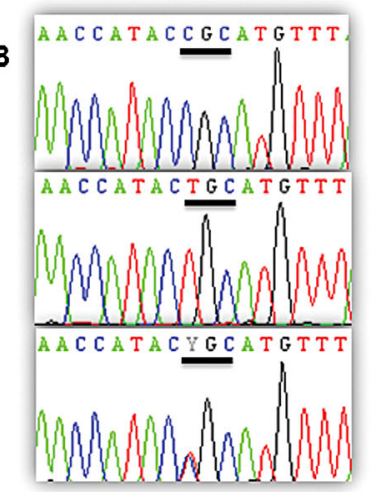

C

H.s. IETSEPYPMFTSR

M.m. LETSEPYFMFTSRV

D.r. HEVTEPYAMFTSA

D.m. IETNEPYFMFTSRA

C.e. LGTNEPYAMFTSRA

s.c. NEVIEPY MPTSRS

s.p. KGVKEPYAVFTSRS

o.s. KDLREPYTMLTSRS

E.c. RGTAEPYFMFTSRA

FIG. 2. Mutations found and amino acid sequence analysis. A: Pedigree showing the segregation of the MT01 c.1510C $>$ T and MT-TF m.593T $>$ G mutations in the family; black symbols indicate affected patients. B: Electropherograms showing the wild-type [top], the homozygous mutated (middle) and heterozygous sequences in MTO1 gene. C: Amino acid sequence alignment from residues $497-510$ of human CCDS34485 reference sequence. The mutated p.R504C in the MT01 protein, squared in red, is fully conserved among eukaryotes and bacteria (Hs: Homo sapiens, Mm: Mus musculus, Dr: Danio rerio, Dm: Drosophila melanogaster, Ce: Caenorhabditis elegans, Sc: Saccaromyces cerevisiae, Sp: Schizosaccharomyces pombe, Os: Oriza sativa, Ec: Escherichia coli]. 
TABLE III. Sequence Variations Found in mtDNA From Leukocytes of Patient II-1

\begin{tabular}{|c|c|c|c|c|}
\hline Nucleotide position & Amino acid substitution & Locus & Presence in Mitomap database & Status \\
\hline$m .263 A>G$ & - & MT-DLOOP & Yes & Polymorphism \\
\hline m.310_311insC & - & MT-DLOOP & Yes & Polymorphism \\
\hline $\mathrm{m} .593 \mathrm{~T}>\mathrm{G}$ & - & MT-TF & No & Mutation \\
\hline $\mathrm{m} .750 \mathrm{~A}>\mathrm{G}$ & - & MT-RNR1 & Yes & Polymorphism \\
\hline m.1438A $>G$ & - & MT-RNR1 & Yes & Polymorphism \\
\hline $\mathrm{m} .3010 \mathrm{G}>\mathrm{A}$ & - & MT-RNR2 & Yes & Polymorphism \\
\hline $\mathrm{m} .4733 \mathrm{~T}>\mathrm{C}$ & p.N88N & MT-ND2 & Yes & Synonymous change \\
\hline$m .4769 A>G$ & p.M100M & MT-ND2 & Yes & Synonymous change \\
\hline$m .8860 A>G$ & p.T112A & MT-ATP6 & Yes & Polymorphism \\
\hline $\mathrm{m} .8950 \mathrm{G}>\mathrm{A}$ & p.V142I & MT-ATP6 & Yes & Polymorphism \\
\hline m.15326A $>G$ & p.T194A & MT-CYB & Yes & Polymorphism \\
\hline m.16189T $>C$ & - & MT-DLOOP & Yes & Polymorphism \\
\hline m.16519T $>C$ & - & MT-DLOOP & Yes & Polymorphism \\
\hline
\end{tabular}

old), the optic atrophy was asymptomatic and in the Patient II-1 (30year-old), the optic atrophy was discovered at 22 years of age, indicating that in adult patients with MTO1 mutations, the optic atrophy is probably moderate. Indeed, the retinal nerve fibre layer around the optic disc showed a decrease in thickness only in the temporal quadrant, a feature of moderate optic neuropathies. One question is whether the optic neuropathy due to MTO1 mutations is progressive? As young patients with MTO1 mutations probably did not have ophthalmic examination, this remains uncertain. Yet, the data from our 30-year-old patient suggest that it is indeed progressing since her vision worsened from 22 to 30 years of age.

In the patients presented herein, the absence of ragged-red fibers was not surprising as the phenotypes were rather mild. Although the muscle histopathology and the serum parameters were also normal, there was an increase in serum lactate reflecting the impairment of the oxidative phosphorylation. Recurrent weakness during the follow-up of both patients might indeed suggest infraclinical episodes of energy failure and lactic acidosis. Decreased activities of complexes I and IV in muscle of the Patient II-1 (4 and $16 \%$ of the mean control values and 8 and $24 \%$ respectively) and the Patient II-2 ( $8 \%$ and $24 \%$ of the mean control values, respectively) were also reported in other patients with MTO1 mutations (7-27\% and 27-35\% for complexes I and IV, respectively) [Baruffini et al., 2013]. Yet, the identification of a mutation in the MT$T F$ gene (Fig. 3A) and the reduced complex I activity in cybrids suggests that this mutation could play a role in the mitochondrial respiratory impairment of the patients. The $\mathrm{tRNA}^{\text {phe }}$ was found mutated in several mitochondrial disorders, including $\mathrm{m} .582 \mathrm{~T}>\mathrm{C}$
A

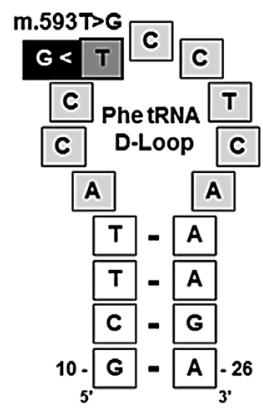

B

\begin{tabular}{|c|c|c|}
\hline 5 & & Polymorphisms / 26850 seq. \\
\hline $14 \cdot$ & $590 \mathrm{~A}$ & \multirow{3}{*}{$\begin{array}{l}A \times 2 \\
A \times 1 ; T \times 3\end{array}$} \\
\hline & $591 \mathrm{C}$ & \\
\hline & 592 C & \\
\hline & $593 \mathrm{~T}$ & G x2; C x100;Ax11; delT x3 \\
\hline & $594 \mathrm{C}$ & \multirow{5}{*}{$\begin{array}{l}T \times 2 \\
C C \times 93 \\
C \times 8 \\
T \times 72 ; C C \times 2 ; C T \times 17 \\
T \times 2\end{array}$} \\
\hline & 595 C & \\
\hline & $596 \mathrm{~T}$ & \\
\hline & $597 \mathrm{C}$ & \\
\hline 22. & 598A & \\
\hline
\end{tabular}

C

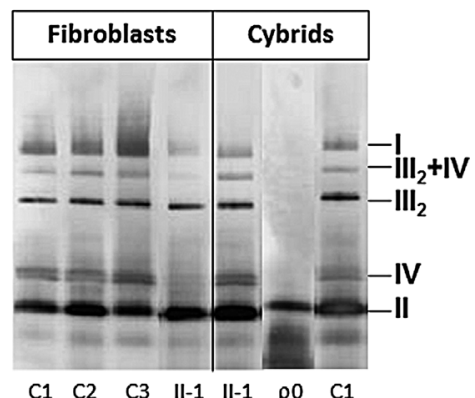

FIG. 3. Structures of the mitochondrial tRNA $A^{\text {phe }} D$-Loop and respiratory chain complexes abundances in fibroblasts and cybrids. A: The sequence variation, $m .593 T>G$ in MT-TF gene, affects an unpaired nucleotide from the loop of the tRNA ${ }^{\text {phe }}$, at the extremity of a base-paired secondary structure. B: Sequence polymorphisms and their occurrence among 26,850 human mitochondrial sequences for each position of the D-Loop of the tRNA ${ }^{\text {phe }}$ are presented and illustrate the exclusion of guanine residue from this structure. C: Protein extracts from fibroblasts and cybrids were loaded on a Blue-Native PAGE gel and transferred on a PVDF membrane, incubated with several antibodies to reveal the RCC complexes, which positions are indicated on the right. C1, C2, and C3 are three normal control fibroblast samples and II-1 corresponds to fibroblasts of patient II-1 showing a drastic reduction of $\mathrm{Cl}$ and $\mathrm{CIV}$. Cybrid extracts from II-1 expressing the m.593T $>G$ mutation show a pattern of RCC comparable to those of a normal individual [C1], while cybrids of rho[0] cells $[\rho 0$ ] show only CII. 
TABLE IV. Respiratory Chain Complex Activities From Fibroblasts and Cybrids Derived From Patient II-1

Ratio of respiration complex activity/citrate synthase activity

\begin{tabular}{|c|c|c|c|}
\hline & \\
\hline & Complex I & Complex II & Complex IV \\
\hline Control fibroblasts & $0.58^{*} \pm 0.18$ & $0.16 \pm 0.02$ & $1.29 \pm 0.24$ \\
\hline Patient fibroblasts & 0.27 & 0.14 & 0.74 \\
\hline Control cybrids & $0.20 \pm 0.02$ & $0.14 \pm 0.08$ & $0.75 \pm 0.36$ \\
\hline Patient cybrids & 0.09 & 0.18 & 0.91 \\
\hline
\end{tabular}

${ }^{*}$ Activities are expressed in nmoles/min/mg of protein and normalized to the citrate synthase activity. Control enzyme activities are given as a mean $+/-$ SD.

and $\mathrm{m} .618 \mathrm{~T}>\mathrm{C}$ in myopathies, $\mathrm{m} .583 \mathrm{G}>\mathrm{A}$ in MELAS, $\mathrm{m} .606 \mathrm{~A}>\mathrm{G}$ in myoglobulinuria, $\mathrm{m} .608 \mathrm{~A}>\mathrm{G}$ in tubule-interstitial nephritis and $\mathrm{m} .611 \mathrm{G}>\mathrm{A}$ in MERRF. Mitochondrial diseases have been shown to result from mutations in mitochondrial genes located in either the nuclear DNA or mitochondrial DNA but synergistic genetic variants in both genomes might also exist in human mitochondrial diseases. For instance, m.593T $>C$ (Fig. 3B), reported as a rare polymorphism in the general population in human mitochondrial databases [Ingman et al., 2006], might impact the onset of LHON in Asian populations [Zhang et al., 2011]. Complex I deficiency was also described in a family with a progressive neurological disorder and a severe muscle complex I enzyme defect, carrying a NDUFA1 mutation in combination with additional mtDNA variants [Potluri et al., 2009]. The m.593T $>$ G transversion reported in this study has been reported so far only twice in mitochondrial databases from a large dataset of mtDNA sequences. It is possible that this change modify the tRNA secondary structure, as guanine residues are excluded from most D-Loops in human mitochondrial tRNAs [Putz et al., 2007]. However, MTO1 is known to catalyzes the 5-carboxymethylaminomethylation of the wobble uridine base in the mitochondrial tRNAs specific to Gln, Glu, Lys, Leu(UUR), and possibly Trp [Wang et al., 2010], but not the tRNA specific to Phe. Therefore, it is unlikely that the MTO1 deficiency caused by the p.Arg504Cys impairs the tRNA ${ }^{\text {phe }}$ function. Yet, since transmitochondrial cybrids containing the patient's mtDNA showed reduced stability of the NDUFA1 subunit and a significant complex I deficiency, it is rather possible that the moderate MTO1 deficiency and the $\mathrm{m} .593 \mathrm{~T}>\mathrm{G}$ transversion had a cumulative effect of the CI activity.

In conclusion, we report the identification of combined MTO1 and $M T-T F$ mutations in two patients with hypertrophic cardiomyopathy, optic neuropathy and cognitive disability. We suggest that further patients with MTO1 mutations should systematically be searched for a moderate optic neuropathy.

\section{ACKNOWLEDGMENTS}

We thank the patients and their family. MC is supported by the Relations Internationales de l'INSERM and SMCT received a fellowship from SOS Rétinite, France. The research was supported by grants for the French Ministry of Health and by the Union Nationale des Aveugles et Déficients Visuels (UNADEV), Retina France, Ouvrir Les Yeux foundations, awarded to CH, GL, and VP.

\section{REFERENCES}

Baruffini E, Dallabona C, Invernizzi F, Yarham JW, Melchionda L, Blakely EL, Lamantea E, Donnini C, Santra S, Vijayaraghavan S, Roper HP, Burlina A, Kopajtich R, Walther A, Strom TM, Haack TB, Prokisch H, Taylor RW, Ferrero I, Zeviani M, Ghezzi D. 2013. MTO1 mutations are associated with hypertrophic cardiomyopathy and lactic acidosis and cause respiratory chain deficiency in humans and yeast. Hum Mutat 34:1501-1509.

Chinnery PF, Turnbull DM. 1997. Clinical features, investigation, and management of patients with defects of mitochondrial DNA. J Neurol Neurosurg Psychiatry 63:559-563.

Chinnery PF, Elliott HR, Hudson G, Samuels DC, Relton CL. 2012. Epigenetics, epidemiology and mitochondrial DNA diseases. Int J Epidemiol 41:177-187.

Ghezzi D, Baruffini E, Haack TB, Invernizzi F, Melchionda L, Dallabona C, Strom TM, Parini R, Burlina AB, Meitinger T, Prokisch H, Ferrero I, Zeviani M. 2012. Mutations of the mitochondrial-tRNA modifier MTO1 cause hypertrophic cardiomyopathy and lactic acidosis. Am J Hum Genet 90:1079-1087.

Ingman M, Gyllensten U. 2006. MtDB: Human Mitochondrial Genome Database, a resource for population genetics and medical sciences. Nucleic Acids Res 34:D749-D751.

Jackson MJ, Schaefer JA, Johnson MA, Morris AA, Turnbull DM, Bindoff LA. 1995. Presentation and clinical investigation of mitochondrial respiratory chain disease. A study of 51 patients. Brain 118:339357.

Koopman WJ, Distelmaier F, Smeitink JA, Willems PH. 2013. OXPHOS mutations and neurodegeneration. EMBO J 32:9-29.

Loiseau D, Chevrollier A, Verny C, Guillet V, Gueguen N, Pou De Crescenzo MA, Ferre M, Malinge MC, Guichet A, Nicolas G, AmatiBonneau P, Malthiery Y, Bonneau D, Reynier P. 2007. Mitochondrial coupling defect in Charcot-Marie-Tooth type 2A disease. Ann Neurol 61:315-323.

Mousson De Camaret B, Taanman JW, Padet S, Chassagne M, Mayencon M, Clerc-Renaud P, Mandon G, Zabot MT, Lachaux A, Bozon D. 2007. Kinetic properties of mutant deoxyguanosine kinase in a case of reversible hepatic mtDNA depletion. Biochem J 402:377-385.

Munnich A, Rotig A, Chretien D, Cormier V, Bourgeron T, Bonnefont JP, Saudubray JM, Rustin P. 1996. Clinical presentation of mitochondrial disorders in childhood. J Inherit Metab Dis 19:521-527.

Nochez Y, Arsene S, Gueguen N, Chevrollier A, Ferre M, Guillet V, Desquiret V, Toutain A, Bonneau D, Procaccio V, Amati-Bonneau P, Pisella PJ, Reynier P. 2009. Acute and late-onset optic atrophy due to a novel OPA1 mutation leading to a mitochondrial coupling defect. Mol Vis 15:598-608. 
Olichon A, Landes T, Arnaune-Pelloquin L, Emorine LJ, Mils V, Guichet A, Delettre C, Hamel C, Amati-Bonneau P, Bonneau D, Reynier P, Lenaers G, Belenguer P. 2007. Effects of OPA1 mutations on mitochondrial morphology and apoptosis: Relevance to ADOA pathogenesis. J Cell Physiol 211:423-430.

Ouhabi R, Boue-Grabot M, Mazat JP. 1998. Mitochondrial ATP synthesis in permeabilized cells: Assessment of the ATP/O values in situ. Anal Biochem 263:169-175.

Potluri P, Davila A, Ruiz-Pesini E, Mishmar D, O'hearn S, Hancock S, Simon M, Scheffler IE, Wallace DC, Procaccio V. 2009. A novel NDUFA1 mutation leads to a progressive mitochondrial complex I-specific neurodegenerative disease. Mol Genet Metab 96:189-195.

Putz J, Dupuis B, Sissler M, Florentz C. 2007. Mamit-tRNA, a database of mammalian mitochondrial tRNA primary and secondary structures. RNA 13:1184-1190.

Rotig A, Lebon S, Zinovieva E, Mollet J, Sarzi E, Bonnefont JP, Munnich A. 2004. Molecular diagnostics of mitochondrial disorders. Biochim Biophys Acta 1659:129-135.

Saks VA, Veksler VI, Kuznetsov AV, Kay L, Sikk P, Tiivel T, Tranqui L, Olivares J, Winkler K, Wiedemann F, Kunz WS. 1998. Permeabilized cell and skinned fiber techniques in studies of mitochondrial function in vivo. Mol Cell Biochem 184:81-100.

Schmiedel J, Jackson S, Schafer J, Reichmann H. 2003. Mitochondrial cytopathies. J Neurol 250:267-277.

Sirvent P, Fabre O, Bordenave S, Hillaire-Buys D, Raynaud de Mauverger E, Lacampagne A, Mercier J. 2012. Muscle mitochondrial metabolism and calcium signaling impairment in patients treated with statins. Toxicol Appl Pharmacol 259:263-268.

Terasaki F, Tanaka M, Kawamura K, Kanzaki Y, Okabe M, Hayashi T, Shimomura H, Ito T, Suwa M, Gong JS, Zhang J, Kitaura Y. 2001. A case of cardiomyopathy showing progression from the hypertrophic to the dilated form: association of Mt8348A- $>$ G mutation in the mitochondrial tRNA(Lys) gene with severe ultrastructural alterations of mitochondria in cardiomyocytes. Jpn Circ J 65:691-694.

Thomas C, Sirvent P, Perrey S, Raynaud E, Mercier J. 2004. Relationships between maximal muscle oxidative capacity and blood lactate removal after supramaximal exercise and fatigue indexes in humans. J Appl Physiol 97:2132-2138.

Tonska K, Kodron A, Bartnik E. 2010. Genotype-phenotype correlations in Leber hereditary optic neuropathy. Biochim Biophys Acta 1797:1119_ 1123.

Wallace DC, Fan W, Procaccio V. 2010. Mitochondrial energetics and therapeutics. Annu Rev Pathol 5:297-348.

Wang X, Yan Q, Guan MX. 2010. Combination of the loss of cmnm5U34 with the lack of s2U34 modifications of tRNALys, tRNAGlu, and tRNAGln altered mitochondrial biogenesis and respiration. J Mol Biol 395:1038-1048.

Zhang AM, Bandelt HJ, Jia X, Zhang W, Li S, Yu D, Wang D, Zhuang XY, Zhang Q, Yao YG. 2011. Is mitochondrial tRNA(phe) variant m.593T $>$ C a synergistically pathogenic mutation in Chinese LHON families with m.11778G $>$ A?. PLoS ONE 6:e26511. 